Feasibility and pilot study of an intervention to support active lifestyles in youth with Type 1

Diabetes: The ActivPals study.

Running title: Lifestyle intervention in youth with T1D

Dr Fiona Mitchell

Chancellor's Fellow/ Lecturer in Physical Activity for Health.

Physical Activity for Health Group

Graham Hills Building,

University of Strathclyde

40 George Street

G1 1QE

Fiona.c.mitchell@strath.ac.uk

(+44) 1415483412 


\section{Feasibility and pilot study of an intervention to support active lifestyles in youth with Type 1}

\section{Diabetes: The ActivPals study.}

Running title: Lifestyle intervention in youth with T1D

Dr Fiona Mitchell*, University of Strathclyde, Glasgow, Physical Activity and Health Group, Scotland. fiona.c.mitchell@strath.ac.uk

Dr Louise Wilkie, University of St Andrews, St Andrews, Scotland. wilkiel@doctors.org.uk

Dr Kenneth Robertson, Children's Diabetes Service, Greater Glasgow and Clyde, Yorkhill Hospital, Glasgow, Scotland, kenneth.robertson1@nhs.net.

Professor John J Reilly, University of Strathclyde, Glasgow, Physical Activity and Health Group, Scotland.John.j.reilly@strath.ac.uk

Dr Alison Kirk, University of Strathclyde, Glasgow, Physical Activity and Health Group, Scotland. Alison.kirk@strath.ac.uk

*corresponding author

Word count: 4300 (excluding refs)

Keywords: Physical Activity, Type 1 diabetes, Youth, Intervention. 


\begin{abstract}
Background: Evidence suggests youth with Type 1 Diabetes (T1D) have lower levels of physical activity (PA) than the general population. The ActivPals intervention aimed to support youth with T1D to lead an active lifestyle.
\end{abstract}

Methods: 20 youth aged 7-16 with T1D were recruited to a pilot randomised controlled trial. PA and Quality of Life (QoL) were measured using Actigraph GT3X+ monitor and Pediatric QoL scales at baseline and one month follow-up. A two way mixed ANOVA showed indicative effects of the intervention. Qualitative interviews were carried out with 16 participants to explore perceptions of the intervention.

Results: An increase in moderate -vigorous PA was reported in intervention and control group from baseline to follow up $(\mathrm{F}(1,14)=5.83 ; \mathrm{p}=0.03)$, with no significant between group differences. Participants in both groups reported significantly less overall diabetes 'problems' $(\mathrm{F}(1,16)=7.93$; $\mathrm{p}=0.012)$ and significantly less lifestyle 'problems' $(\mathrm{F}(1,16)=7.39 \mathrm{p}=0.015)$ at follow up. However, both groups also reported significant increases in 'problems' with the day to day diabetes routine (F $(1,16)=6.48 ; \mathrm{p}=0.022)$ at follow up. Parents reported significant increased worry about their child's diabetes at follow up, in both groups $(F(1,14)=5.83 ; p=0.046)$. There was no significant increase in reported hypoglycaemic occurrences despite increased PA. The qualitative data highlights that goalsetting, self-monitoring and social support were effective motivators for increasing PA.

Conclusions: A larger trial with longer follow up should be conducted to explore the effect of the intervention on PA in youth with T1D.

This study was funded by Yorkhill Children's Charity. 


\section{Introduction}

Regular physical activity (PA) has many physical and psychological health benefits for individuals with Type 1 diabetes (T1D) and is recommended in current clinical guidelines (1). Regular PA may reduce the risk of developing diabetes related health risks and complications (e.g retinopathy, nephropathy, cardiovascular disease and cerebrovascular disease $(2,3)$ and is associated with a better quality of life $(\mathrm{QoL})(4,5)$. Increasing QoL is of particular importance as youth with T1D have reported poorer quality of life (QoL) than youth without T1D $(6,7)$. Despite the clear benefits of regular PA, youth with T1D lead less active lives $(8-10)$, and have poorer health outcomes $(11,12)$ than youth without T1D. PA levels in this population are also well below the recommendations for health, with around two thirds of youth with T1D failing to meet the target of 60 minutes per day of moderate to vigorous physical activity (MVPA) and spending prolonged periods of time sedentary (10). Designing effective interventions to support PA in youth with T1D is an important area of research. While PA intervention development in this population is growing, methodological limitations of previous work make it difficult to assess intervention effectiveness. For example, typically studies are not based on behavioural change theories $(13,14)$, have uncontrolled designs $(15$, 16), or consist of a very structured supervised intervention design (e.g. using supervised structured exercise classes in the intervention) (16-19). Whilst supervised settings may result in short-term changes in PA, these changes are often not maintained post intervention (20).

The MRC framework for developing and evaluating complex interventions strongly advises carrying out feasibility and pilot work prior to running a full-scale trial; therefore, in keeping with phases 1 and 2 of the MRC framework, the aim of this study was to use a mixed methods study design to determine: 
1. The recruitment, initial retention and adherence levels that can be achieved for a four-week intervention programme in both the intervention and control groups.

2. Preliminary evidence of effect of the intervention on physical activity, sedentary behaviour and quality of life.

3. Participants' perceptions of the intervention, for supporting an active lifestyle.

\section{ActivPals intervention}

The ActivPals intervention aimed to: support youths with T1D to initiate and maintain an active lifestyle, including increased MVPA and reduced sedentary behaviour. The intervention includes a physical activity consultation (21), based on Social Cognitive Theory (22). This consultation model has been successfully used with other diabetes groups (21). The intervention was designed by the research team based on existing evidence and consultations with young people with T1D, parents and health professionals working with youth with T1D. Strategies and techniques identified as important for supporting behaviour change were incorporated in the consultation to support initiation and maintenance of an active lifestyle. This was focused on increasing motivation and reducing barriers to physical activity, with additional discussion of self-efficacy, decisional balance and techniques to support behaviour change. Goal setting was used to agree a four-week individualised graduated PA programme, in the form of a specially designed diary booklet. Participants were encouraged to record daily steps in this diary. A motivational video message from an athlete with T1D was also provided to participants. In addition, a self-monitoring pedometer wrist device which records daily steps and syncs to a mobile app and website, was given to all participants in the intervention group. The researcher prompted the participants to adhere to their individualised physical activity plan via text message, throughout the four- week intervention period. A nominated parent supported each young person throughout the full intervention period. The ActivPals intervention was tailored to the individual's baseline activity, activity preferences and local opportunities. The intervention was delivered by the researcher (first author) who is collecting the data for the study. More information on 
the ActivPals intervention can be found in the protocol paper (23). The physical activity consultation booklets that were used with young people and parents during the consultation are provided as an appendix (appendix 1 and 2 respectively). 


\section{Methods}

\section{Recruitment}

A full recruitment strategy is provided as an appendix in the protocol paper (23). Recruitment of participants took place between January and March 2016 and finished when the target sample size was reached $(n=20)$. The sample size was based on recommendations from authors specialising in feasibility and pilot studies $(24,25)$. The sample will introduce sufficient variance to examine the feasibility of a larger study. Participants were recruited to the study from two points 1) paediatric diabetes clinics (main recruitment site) and (2) through new start groups for young people with T1D. Participants who were eligible and interested in participating in the study were given an information pack with details about participation. The researcher then contacted participants and arranged a visit to discuss the study. Participants were eligible if they were aged 7-16 with a medical diagnosis of T1D, were registered in Greater Glasgow and Clyde (GG \& C) Children's Diabetes Service and were independently ambulatory.

\section{Quantitative outcome measures}

To examine preliminary effectiveness of the four-week PA intervention, ActivPals, objectively measured PA (light and moderate to vigorous physical activity (MVPA)) sedentary behaviours, body mass index and quality of life data were collected. Details of these measures are provided below. Outcomes were collected at baseline (pre-intervention/pre-control) and four-week post intervention/post control follow up.

\section{Randomisation}

After baseline measures were collected, the PI of the study (XX) used computer software to randomly allocate participants to an intervention group or a waiting list control group. Allocation of group was 
concealed from the researcher who was collecting the data and delivering the intervention (XX), until immediately before the intervention/control group visit. Those allocated to the control group were offered the intervention once all post-control data had been collected. The study protocol is described in full elsewhere (23). Full NHS ethical approval was been granted for the study by the appropriate research ethics committee.

\section{Accelerometer data}

Objective measures of physical activity and sedentary behaviour were collected using the Actigraph GT3X + monitor. This monitor allows objective recording of daily time spent in sedentary, light and moderate to vigorous physical activity. These monitors are small (approx. size of a UK £2 coin) and lightweight (19 g). Participants were asked to wear the accelerometers around the waist during waking hours for 7 days, excluding water-based activities. Accelerometer data was downloaded to Actilife software (version 6.4.3). In line with previous studies, a minimum wear time for a valid day was defined as $6 \mathrm{~h}$ /day, with a minimum of 3 days of data required for analysis inclusion $(26,27)$. The primary outcome measure of $\%$ of daily time spent in MVPA and sedentary behaviour was determined using cut-points calibrated and validated in paediatric studies: sedentary $(<100 \mathrm{cpm})(28)$ and MVPA ( $\geq 3200 \mathrm{cpm})$ (29). A macro was used to calculate average time worn per day. This was designed by an expert in accelerometer data who has developed and tested this extensively with various populations (26). To ensure that sleep data was not included in the analysis, data recorded between 12:00 -6:00am was excluded from the analysis, for all participants (unless otherwise reported in wear time diaries). This was consistent with previous research with this population (30). The researchers also checked the data visually to check if any participants that had worn the accelerometer overnight slept past 6am. In such cases, this was manually adjusted in the macro and removed until the participant was awake.

Periods of consecutive zeros, other than that recorded in wear diaries as sleep time or non-wear, were kept in the data, as assumptions were not made to define periods as non-wear or sedentary behaviour. 
All participants wore the accelerometer for at least 6 hours a day for at least 3 days a week, therefore all were included in the analysis. If accelerometers were worn for less than 7 days, average time in PA and sedentary behaviour was adjusted and calculated for each valid day. In total, full accelerometer data was analysed for 16 participants, 8 in each arm of the intervention.

\section{Quality of Life questionnaires}

Generic and disease-specific questionnaires were used to measure QoL in participants. The PedsQoL 4.0 Generic Core Scale was used to measure general quality of life (31). This 23-item questionnaire contains the following subscales: physical functioning, emotional functioning, social functioning and school functioning. A psychosocial health summary score was calculated from the average of the emotional, social and school functioning subscales, a physical health summary score from the physical functioning subscale and a total overall score from the average of all subscales. This scale has shown good reliability and validity in this population $(31,32)$. The PedsQoL 3.0 Type 1 Diabetes Module is a 28-item questionnaire measuring diabetes-specific QoL and consists of five subscales: diabetes symptoms, treatment barriers, treatment adherence, worry and communication. Patients (selfreport) and their parents (proxy-report of the child's QoL) completed questionnaires by rating items on how much each was a problem in the previous month using a 5-point Likert scale (' 0 ' = never a problem; '4' = almost always a problem). This questionnaire has been validated and has been shown to be reliable in youth with Type 1 Diabetes $(31,33)$. Changes in general QoL and diabetes module scores were analysed between intervention and control groups to asses for any trends in intervention effects.

\section{Anthropometric measures}

Participants were invited to have their weight, height and waist circumference measured wearing light clothes without shoes. All measurements were made in duplicate and the final value calculated as the 
mean of the two measurements. Weight in kilograms $(\mathrm{kg})$, was measured to the nearest $100 \mathrm{~g}(\mathrm{~g})$, using SECA 877 scales (SE approval class III; SEA Germany). Height in metres (m) was measured to the nearest $1 \mathrm{~mm}(\mathrm{~mm})$ using the SECA Leicester stadiometer (SECA, Germany). The height $(\mathrm{m})$ and weight $(\mathrm{kg})$ were used to calculate BMI using the formula; BMI $=$ weight $/$ height $^{2}(\mathrm{~kg} / \mathrm{m} 2)$. Waist circumference was measured to the nearest $0.5 \mathrm{~cm}(\mathrm{~cm})$ at the mid-point between the iliac crest and the lowest rib, in full expiration with the participant standing.

\section{Qualitative interviews}

Semi- structured qualitative interviews were carried out with $16 / 20$ of the study, as four participants dropped out of the study before the interviews were completed. Interviews were carried out from May to July 2016 with seven male and nine female participants (mean age of $11.6 \pm 2.5$ years). Age at diagnosis ranged from 1 to 13 years (mean $7.8 \pm 4.0$ years) while duration time from diagnosis ranged from 3 months to 12 years $(3.8 \pm 4.3$ years). Seven participants were relatively newly diagnosed $(\leq 6$ months). 10 were treated with insulin injections, while 6 were pump users. A parent was also included in the interviews. Interviews were carried out at participants' homes and lasted around 30 minutes. The interviewer was a GP with T1D undertaking an MSc in Sports Medicine, and was not directly involved with delivery of the ActivPals intervention. Participants were made fully aware of this prior to interview. Participant and parents were recruited after they had completed the 4 week ActivPals PA intervention, and provided written and verbal consent to be interviewed. Interviews were recorded, transcribed verbatim and analysed thematically. This paper presents one key theme identified from the qualitative data; components of the intervention perceived as most effective in supporting an active lifestyle. A comprehensive qualitative paper exploring motivations for physical activity participation for youth with T1D has been published separately (34). 


\section{Data Analysis}

\section{Statistical Analysis}

As this was a feasibility and pilot study, quantitative outcomes provide preliminary evidence of effect of the intervention on PA, sedentary behaviour and quality of life. The results of this study will allow the authors to calculate the sample size required for a future full- scale trial. Statistical analysis was carried out using SPSS. An intention to treat approach was used for the analyses, with all participants analysed in the group to which they were randomised. The PA outcome data assessed change in percentage of waking day time spent in light and MVPA and percentage of time spent sedentary, at four weeks from baseline. In addition, changes in quality of life (QoL) general and diabetes specific, in youth and parents, were analysed at four weeks from baseline. These was analysed at the level of the individual, using a two way mixed ANOVA. Descriptive statistics are presented below (mean and standard deviation) with $95 \%$ confidence intervals presented for each group separately.

\section{Qualitative analysis}

Interviews were recorded, transcribed verbatim and analysed using a six-stage thematic process (35). Transcription by the interviewer allowed familiarisation with the data. The researcher who carried out the interviews read the transcripts several times before identifying initial themes. Transcripts were reread and themes and subthemes refined. To enhance reliability, $20 \%$ of the transcripts were systematically coded independently by the first author (XX), who also designed the interview guide The researchers searched for consistent patterns of meanings and relationships across transcripts and grouped categories together, as well as noting divergent views. This process was used until data saturation was achieved. 


\section{Results}

\section{Recruitment, retention and adherence to study}

The goal of recruiting 20 young people with T1D was achieved by recruited from T1D clinic and groups. 10 were randomised to the ActivPals intervention ( $\mathrm{n}=7$ females) and 10 to the waiting list control group ( $\mathrm{n}=5$ females). The mean age of participants in both groups was 12 years. In total, valid accelerometer data was available for $16(80 \%)$ of the 20 participants at the four- week data collection point (Fig. 1). The proportion of participants lost to follow up was the same for the ActivPals (10\%) and control group (10\%) and there were no differences in baseline characteristics between participants lost to follow up and completers.

\section{Preliminary evidence of changes in PA, sedentary behaviour and QoL}

There was no significant post-intervention effects on $\%$ of waking time spent in light PA or $\%$ of time spent sedentary (within or between groups) at four weeks (see table 1). While there was no significant intervention effect (between group difference) in \% of time spent in MVPA, results showed a significant increase in \% of time in MVPA in both intervention and control group from baseline to follow up $(\mathrm{F}(1,14)=5.83 ; \mathrm{p}=0.03)$.

For QoL measures, participants in both groups reported significantly less overall diabetes 'problems' $(\mathrm{F}(1,16)=7.93 ; \mathrm{p}=0.012)$ and significantly less lifestyle 'problems' $(\mathrm{F}(1,16)=7.39 \mathrm{p}=0.015)$ at follow up. However, both groups also reported significant increases in 'problems' with the day to day diabetes routine $(F(1,16)=6.48 ; \mathrm{p}=0.022)$ at follow up. Parents reported significant increased worry about their child's diabetes at follow up, in both groups $(\mathrm{F}(1,14)=5.83 ; \mathrm{p}=0.046)$. There was no significant increase in reported hypoglycaemic occurrences despite increased MVPA. 


\section{Participants' perceptions of the intervention, for supporting an active lifestyle.}

The ActivPals PA intervention included a range of behaviour change techniques including goal setting, self- monitoring (using a pedometer and app), action planning, social support and role modelling. Generally these components were viewed favourably by participants and parents, suggesting the intervention was acceptable and viewed positively by the study population it was developed for. The intervention components perceived as most 'effective' were goal- setting, selfmonitoring and social support (received mainly from parents and the researcher).

\section{Goal setting, self-monitoring and social support}

Goal setting was a key technique used in the intervention and almost all of the participants felt that setting goals encouraged their participation. In particular, making a plan and writing down activities for 4 weeks in the planner diary was viewed as motivating:

"If we didn't have it written down you might have gone 'don't think I'll bother today'." (Mum of P5, $\mathrm{M} ; 7)$

The pedometer used in the study (linked to a smart phone app) was universally acknowledged to provide motivation to increase PA. This tracked daily steps, so participants were able to self- monitor if they were meeting the goals they set with the researcher;

"It taught me to do the best I could and to motivate myself because if I looked and saw I hadn't done that many steps then I could go and get out and do something, so it motivated me to do more." (P8, $\mathrm{F} ; 12)$

While there were some technical and practical challenges reported about the pedometer device (mainly that it could easily be lost and did not always charge effectively), many of the participants talked about the pedometer as a fun 'gadget' to show their progression and compete with others. For a few participants, this may have been the motivating factor to take part in the study: 
"I think he is quite open to these technical things so in my opinion, it was the reason that he decided to take part." (Dad of $\mathrm{P} 9, \mathrm{M} ; 8)$

Social support for living an active lifestyle was identified as crucial in this intervention. Parental support varied from providing words of encouragement, driving children to activities, to physically participating in activities with their children. Interestingly, many parents were keen to use a pedometer so they could also monitor their own steps and participate in physical activity with their child. The involvement of parents often differed with age of participant, with younger participants often being more keen to exercise with parents:

"And I like walking more with my mum because that's encouraging me to do lots more stuff and do more running about" (P2, M;10)

In addition to parental support, the support of a health professional was also valued. In this case, the researcher who carried out the intervention encouraged participants throughout the intervention period. This external social support was viewed favourably, particularly by the older (adolescent) participants in the study. This move into adolescence often results in young people seeking independence from their parents; therefore an external support was particularly useful in such cases:

“...suggestions from someone who does that for a living...it helps to see the bigger picture and kind of recommend more things to do" $(\mathrm{P} 12, \mathrm{~F} ; 13)$ 


\section{Discussion}

Despite no significant difference between the intervention and control group for increased PA or reduced sedentary time over a four-week individualised intervention, the results suggest that both intervention and control groups significantly increased their MVPA. This is an interesting finding and suggests that participating in a PA research study may, in itself, have motivated youths to increase their MVPA. As participants were either newly diagnosed with the condition or were attending a T1D clinic appointment when they were recruited, there may have been an increased readiness to change behaviour, for some participants. In addition, it may be that seasonal changes (participants were recruited from January- March) also played a role in increased PA in the participants. As this study was carried out in Scotland, winter can be particularly cold with early dark nights. Recruitment to this study from January may have motivated participants to begin to get active as the spring months approached, suggested by previous Scottish studies (36). A larger scale study would include a longer recruitment period and therefore recruitment could be mapped to seasons to assess any differences.

There was no significant difference between the intervention and control group for total QoL scores at baseline and follow up. However, both the intervention and control group had significantly higher overall QoL scores on the child/teenage questionnaire at follow up, from baseline. A higher score indicates less diabetes 'problems' and greater quality of life, therefore this is a positive finding. Interestingly, there was a significant increase in problems reported in treatment 1 subscale of the diabetes questionnaire and a significant increase in child report scores of treatment 2 of diabetes in both intervention and control group. Treatment subscale 1 asks questions 'It hurts to prick my finger or give myself insulin injections', 'I am embarrassed about having diabetes', 'my parents and I argue about my diabetes care' and 'it is hard for me to stick to my diabetes routine'. The significant change in scores from baseline to follow up, in both groups, shows that there were more 'problems' with treatment. This may relate to the increased MVPA levels seen in participants, which is likely to have resulted in increased self-monitoring of their diabetes (37). For example, more finger prick tests needed, more adjustments for increased PA and increased challenges with sticking to their diabetes 
routine. Therefore, the positive change in MVPA may also have resulted in increased challenges of some aspects of managing diabetes, which is supported by previous work (38).

Interestingly, in the treatment 2 subscale the young people rated their problems for treatment of diabetes significantly lower from baseline to follow up ('it is hard for me to do blood glucose tests', 'it is hard for me to give myself insulin shots', 'it is hard for me to exercise', 'it is hard for me to follow a healthy diet', 'it is hard for me to wear an id bracelet/carry a card', 'it is hard for me to carry a fast acting carbohydrate', 'it is hard for me to eat snacks between meals when I should'). This suggests that practicing the behaviours of good diabetes management was significantly better at follow up than baseline (exercising, following a healthy diet, eating snacks when should).

The results also indicate a significant increase in parents reported worry about their child's diabetes from baseline to follow up in both groups. This may also be related to the increased MVPA, as parents were aware that there was more self- monitoring and management needed. Parents worry may have increased as young people's problems with diabetes treatment increased (treatment 1 subscale). Previous research has highlighted that more psychosocial support is needed for parents as they adjust to a new diagnosis of T1D and through changes in their child's lifestyle (39). The future trial will include a focus on providing psychosocial support to parents and youth.

The qualitative data suggests that nearly all of our participants felt that having goals encouraged their participation, with most of those in favour of comparing daily steps with family members or friends. The pedometer therefore provided a competitive aspect to their behaviour. The use of a pedometer was universally acknowledged to provide motivation through self-monitoring and goal setting and was an incentive for participation to the more technologically minded children. Future research with this population could develop technologies further to incorporate PA, dietary and blood sugar monitoring, within one device. The type of social support required is likely to be age related, therefore future interventions should ensure a suitable family and/or external support is available to participants. 
This study provides interesting findings about the recruitment, retention and indicative results of a four- week PA intervention for youth with Type 1 Diabetes. These results suggest taking part in PA research study may have potential to increase MVPA levels and overall QoL in youth with T1D without significantly increasing hypoglycaemic episodes. While there was increased parental worry and increased treatment problems (subscale 1), this was likely to be a reaction to the short- term change in lifestyle behaviours and resultant changes in blood glucose. We suggest that a full- scale intervention study which has a parental psychosocial support component would be an effective strategy to combat this. In addition, as PA increases over a longer period and lifestyle changes became part of daily living, the perceived worry associated with change of routine would reduce.

\section{Limitations of the study}

As this was a small scale trial, with the researcher delivering the intervention (therefore not blind to the trial), we want to emphasise that the results show indicative effects, rather than definitive results. The PA and QoL findings are interesting and suggest the need for a larger scale trial, with a longer intervention period and longer term post-intervention follow up data. The full scale intervention will build on the results of the pilot and feasibility work and take into account the feedback from the qualitative interviews. For example, we plan to do a separate project which focusses on developing technology for increasing healthy lifestyles in youth with type 1 diabetes. This will then provide tailored and a more sophisticated device and app to address many of the challenges faced in this pilot study. 


\section{Safety and adverse events}

There were no adverse events reported from this study.

\section{Acknowledgements}

We would like to thank Storm Health for providing monitoring devices and use of the Get Active device and platform. 
1. Standards of Medical Care in Diabetes-2016 Abridged for Primary Care Providers. Clinical Diabetes. 2016;34(1):3-21.

2. MacMillan F, Kirk A, Mutrie N, Matthews L, Robertson K, Saunders DH. A systematic review of physical activity and sedentary behavior intervention studies in youths with type 1 diabetes: study characteristics, intervention design, and efficacy. Pediatr Diabetes. 2014;15.

3. Quirk H, Blake H, Tennyson R, Randell TL, Glazebrook C. Physical activity interventions in children and young people with Type 1 diabetes mellitus: a systematic review with meta-analysis. Diabetic Medicine. 2014;31(10):1163-73.

4. Gopinath B, Hardy LL, Baur LA, Burlutsky G, Mithell P. Physical activity and sedentary behaviors and health-related quality of life in adolescents. Pediatrics. 2012;130(1):e167-74.

5. Shoup JA, Gattshall M, Dandamudi P, Estabrooks P. Physical activity, quality of life and weight status in overweight children. Qual Life Res. 2008;17(3):407-12.

6. Delamater AM. Quality of life in youths with diabetes. Diabetes Spectr. 2000;13(1):42-7.

7. Kalyva E, Malakonaki E, Eiser C, Mamoulakis D. Health related quality of life ( $\mathrm{HrQoL})$ of children with type 1 diabetes mellitus (T1DM): Self and parental perceptions. Pediatric Diabetes. 2011;12(1):34-40.

8. Maggio A, Hofer MF, Martin X, Marchand L, Beghetti M, Farpour-Lambert N. Reduced physical activity level and cardiorespiratory fitness in children with chronic diseases. Eur J Pediatr. 2010;169.

9. Valerio G, Spagnuolo M, Lombardi F, Spadaro R, Siano M, Franzese A. Physical activity and sports participation in children and adolescents with type 1 diabetes mellitus. Nutr Metab Cardiovasc Dis. 2007;17.

10. MacMillan F, Kirk A, Mutrie N, Robertson K. Physical activity and sedentary behaviour in Scottish youth with type 1 diabetes. Practical Diabetes. 2014;31(6):228-33c.

11. Kalyva E, Malakonaki E, Eiser C, Mamoulakis D. Health related quality of life (HrQoL) of children with type 1 diabetes mellitus (T1DM): self and parental perceptions. Pediatr Diabetes. 2011;12.

12. Wennick A, Hallström I, Lindgren B, Bolin K. Attained education and self-assessed health later in life when diagnosed with diabetes in childhood: a population-based study. Pediatr Diabetes. 2011;12.

13. MacMillan F, Kirk A, Mutrie N, Matthews L, Robertson K, Saunders DH. A systematic review of physical activity and sedentary behavior intervention studies in youth with type 1 diabetes: study characteristics, intervention design, and efficacy. Pediatric Diabetes. 2014;15(3):175-89.

14. Newton KH WE, Elley CR. Pedometers and Text Messaging to Increase Physical Activity Randomized controlled trial of adolescents with type 1 diabetes. Diabetes Care. 2009;32:813-5.

15. Faulkner M, Fleet, S., Hepworth, J. A personalized approach to exercise promotion in adolescents with type 1 diabetes. Pediatric Diabetes. 2010;11:166-74.

16. Mosher PE, Nash MS, Perry AC, LaPerriere AR, Goldberg RB. Aerobic circuit exercise training: effect on adolescents with well-controlled insulin-dependent diabetes mellitus. Arch Phys Med Rehabil. 1998;79.

17. Tunar M, Ozen S, Goksen D, Asar G, Bediz CS, Darcan S. The effects of Pilates on metabolic control and physical performance in adolescents with type 1 diabetes mellitus. J Diabetes Complications. 2012;26.

18. Salem M, AboElAsrar MA, Elbarbary N, ElHilaly R, Refaat $\mathrm{Y}$. Is exercise a therapeutic tool for improvement of cardiovascular risk factors in adolescents with type 1 diabetes mellitus? $A$ randomised controlled trial. Diabetol Metab Syndr. 2010;2.

19. Stratton R, Wilson DP, Endres RK, Goldstein DE. Improved glycemic control after supervised 8-wk exercise program in insulin-dependent diabetic adolescents. Diabetes Care. 1987;10.

20. Biddle S, Mutire, N., Gorley, T. Psychology of Physical Activity. 3 ed. Oxon: Routledge 2015. 
21. Kirk A, Barnett J, Mutrie N. Physical activity consultation for people with type 2 diabetes. Evidence and guidelines. Diabet Med. 2007;8:809 - 16.

22. Bandura A. Social foundations of thought and action: a social cognitive theory. New Jersey: Prentice-Hall; 1986.

23. Mitchell F, Kirk A, Robertson K, Reilly JJ. Development and feasibility testing of an intervention to support active lifestyles in youths with type 1 diabetes-the ActivPals programme: a study protocol. Pilot and Feasibility Studies. 2016;2(1):66.

24. Lancaster G, Dodd S, Williamson P. Design and analysis of pilot studies: recommendations for good practice. J Eval Clin Practice. 2004;10:307-12.

25. Browne R. On the use of a pilot sample for sample size determination. stat med. 1995;14:1933-40.

26. Janssen X, Basterfield, L., Parkinson, K., Pearce, M, Reilly, J., Adamson, A., Reilly, J. Objective measurement of sedentary behaviour: impact of non-wear time rules on changes in sedentary time. BMC Public Health. 2015;15(504).

27. Basterfield L, Adamson, A.J., Pearce, MS., Reilly, JJ. Stability of habitual physical activity and sedentary behaviour monitoring by accelorometry in 6 to 8 year olds. journal of physical activity and health. 2011;8:543-7.

28. Evenson KR, Catellier DJ, Karminder G, Ondrak KS, McMurray RG. Calibration of two objective measures of physical activity for children. J Sports Sci. 2008;24.

29. Puyau MR, Adolph AL, Vohra FA, Butte NF. Validation and calibration of physical activity monitors in children. Obes Res. 2002;10.

30. MacMillan F, Kirk A, Mutrie N, Robertson K. Physical activity and sedentary behaviour in Scottish youths with type 1 diabetes. Pract Diabetes. 2014;31.

31. Varni JW, et al. The PedsQL in type 1 and type 2 diabetes: reliability and validity of the Pediatric Quality of Life Inventory Generic Core Scales and type 1 Diabetes Module. Diabetes Care. 2003;26(3):631-7.

32. Upton $\mathrm{P}$, et al. Measurement properties of the UK-English version of the Pediatric Quality of Life Inventory 4.0 (PedsQL) generic core scales. . Health and Quality of Life Outcomes, . 2005;3:2.

33. Sand $P$, et al. The reliability of the health related quality of life questionniare PedsQL 3.0 Diabetes ModuleTM for Swedish children with type 1 diabetes Acta Paediatrica, . 2012;101(8):e344e9.

34. Wilkie L, Mitchell F, Robertson K, Kirk A. Motivations for physical activity in youth with type 1 diabetes participating in the ActivPals project: a qualitative study. Practical Diabetes. 2017;34(5):151-5.

35. Braun V, Clarke V. Using thematic analysis in psychology. Qual Res Psychol. 2006;3.

36. Fisher A, Reilly JJ, Montgomery C, Kelly LA, Williamson A, Jackson DM, et al. Seasonality in Physical Activity and Sedentary Behavior in Young Children. Pediatric Exercise Science.

2005;17(1):31-40.

37. Valerio G SM, Lombardi F, Spadaro R, Siano M, Franzese A. . Physical activity and sports participation in children and adolescents with type 1 diabetes mellitus. . Nutrition, metabolism and cardiovascular diseases. 2007;17(5):376-82.

38. Metcalf KM, Singhvi A, Tsalikian E, Tansey MJ, Zimmerman MB, Esliger DW, et al. Effects of Moderate-to-Vigorous Intensity Physical Activity on Overnight and Next-Day Hypoglycemia in Active Adolescents With Type 1 Diabetes. Diabetes Care. 2014;37(5):1272-8.

39. Hilliard ME, Tully C, Monaghan M, Wang J, Streisand R. Design and development of a stepped-care behavioral intervention to support parents of young children newly diagnosed with type 1 diabetes. Contemporary Clinical Trials. 2017;62:1-10. 


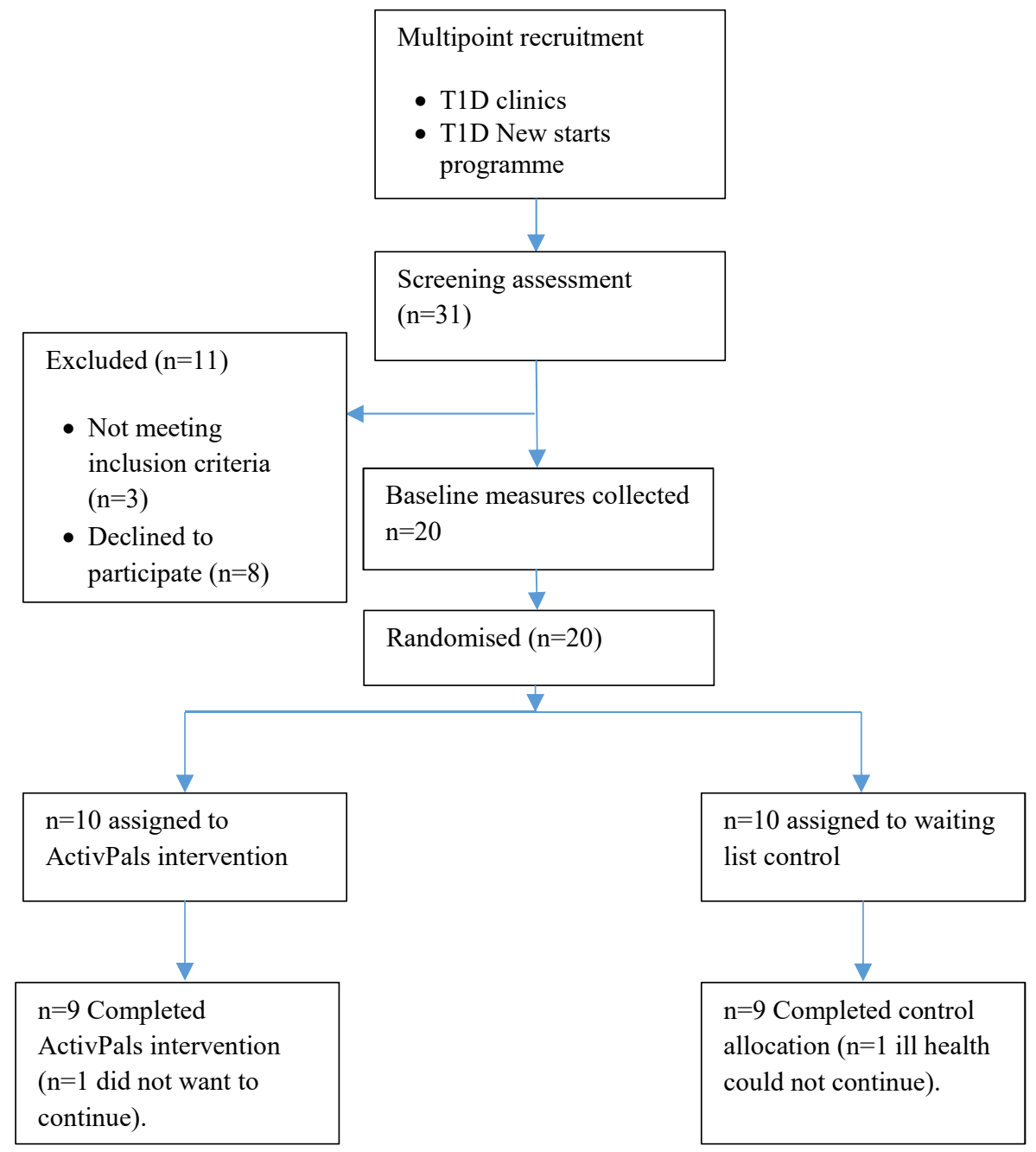

Figure 1. Recruitment, initial retention and adherence level of Activpals 4- week intervention 
Table 1. Baseline, follow up and differences in PA and QoL outcomes

\begin{tabular}{|c|c|c|c|c|c|c|}
\hline Outcomes & \multicolumn{2}{|c|}{$\begin{array}{l}\text { Baseline } \\
(n=) \text { Mean (SD) }\end{array}$} & \multicolumn{2}{|c|}{$\begin{array}{l}4 \text { week follow up } \\
\text { Mean (SD) }\end{array}$} & \multicolumn{2}{|c|}{$\begin{array}{l}\text { Difference in mean score from } \\
\text { baseline to } 4 \text { weeks (baseline-4 } \\
\text { weeks follow up) ( } 95 \% \mathrm{Cl})\end{array}$} \\
\hline PA measures & $\begin{array}{l}\text { Intervention } \\
(n=8)\end{array}$ & Control $(n=8)$ & $\begin{array}{l}\text { Intervention } \\
(n=8)\end{array}$ & $\begin{array}{l}\text { Control } \\
(n=8)\end{array}$ & Intervention & Control \\
\hline Minutes LPA & $254.0(86.1)$ & $253.1(77)$ & $219.1(83.5)$ & $218.1(57.5)$ & $-34.9(-11.3-81.4)$ & $-35(-11.3-81.3)$ \\
\hline$\%$ LPA & $23.8(7.9)$ & $23.4(7.1)$ & $20.7(8)$ & $20.8(5.2)$ & $-3.1(-1.2-7.3)$ & $-2.6(-1.6-6.9)$ \\
\hline Minutes MVPA & $18.2(16)$ & $22.5(16.2)$ & $24.7(14.1)$ & $38(22.1)$ & $6.5(-20.9-8.0)$ & $\begin{array}{l}15.5(-29.9--1.0 \\
)\end{array}$ \\
\hline$\%$ MVPA & $1.7(1.5)$ & $2.1(1.5)$ & $2.3(1.3)$ & $3.6(2.0)$ & $0.6(-1.9-0.7)$ & $1.59(-2.8-0.2)$ \\
\hline $\begin{array}{l}\text { Minutes } \\
\text { Sedentary }\end{array}$ & $797.7(88.4)$ & $798.6(69.6)$ & 819.9 (98.9) & $791.5(66.7)$ & $22.2(-71.1-26.8)$ & $-7.1(-42.3-55.6)$ \\
\hline$\%$ sedentary & $74.5(8.5)$ & $74.4(7.2)$ & $76.9(8.6)$ & $75.6(7.1)$ & $2.4(-7-2.2)$ & $1.2(-5.7-3.5)$ \\
\hline $\begin{array}{l}\text { QoL overall } \\
\text { measures }\end{array}$ & $\begin{array}{l}\text { Intervention } \\
(n=9)\end{array}$ & Control $(n=9)$ & $\begin{array}{l}\text { Intervention } \\
(n=9)\end{array}$ & $\begin{array}{l}\text { Control } \\
(n=9)\end{array}$ & Intervention & Control \\
\hline $\begin{array}{l}\text { QoL general } \\
\text { child/teenage }\end{array}$ & $81.4(10)$ & $76.4(15)$ & $81.5(11.2)$ & $81.2(13)$ & $0.2(-4.8-4.6)$ & $4.8(-9.4-0)$ \\
\hline $\begin{array}{l}\text { QoL general } \\
\text { parent }\end{array}$ & $79.1(8.4)$ & $75(14)$ & $79.1(11)$ & $79(14)$ & $0(9-6.8-6.8)$ & $4(-11-2.5)$ \\
\hline $\begin{array}{l}\text { QoL diabetes } \\
\text { specific } \\
\text { child/teenage }\end{array}$ & $67(12.4)$ & $69.2(12.3)$ & $72.3(12.1)$ & $74(11)$ & $5.3(-11.2-0)$ & $4.8(-10.4-0.7)$ \\
\hline $\begin{array}{l}\text { QoL diabetes } \\
\text { specific parent }\end{array}$ & $68(15)$ & $72.6(10.5)$ & $71(12)$ & $73(12)$ & $3(-7.7-2)$ & $0.4(-4.7-4.9)$ \\
\hline
\end{tabular}

